\title{
Philosophiques
}

\section{Collectif. Philosophie et littérature. Coll. « L’univers de la philosophie », Montréal, Bellarmin, 1979, 189 p.}

\section{Robert Hébert}

Volume 10, numéro 1, avril 1983

URI : https://id.erudit.org/iderudit/203223ar

DOI : https://doi.org/10.7202/203223ar

Aller au sommaire du numéro

Éditeur(s)

Société de philosophie du Québec

ISSN

0316-2923 (imprimé)

1492-1391 (numérique)

Découvrir la revue

Citer ce compte rendu

Hébert, R. (1983). Compte rendu de [Collectif. Philosophie et littérature. Coll.

"L'univers de la philosophie ", Montréal, Bellarmin, 1979, 189 p.]

Philosophiques, 10(1), 188-191. https://doi.org/10.7202/203223ar d'utilisation que vous pouvez consulter en ligne.

https://apropos.erudit.org/fr/usagers/politique-dutilisation/ 
Collectif. Philosophie et littérature. Coll. "L'univers de la philosophie ", Montréal, Bellarmin, 1979, 189 p.

\section{par Robert Hébert}

Les rapports entre la philosophie et la littérature ont depuis toujours fasciné philosophes et littéraires, plus encore ceux qui poursuivent jusquà ses limites visibles une pratique poétique de l'écriture. Les uns et les autres ayant parfois le loisir de cerner à la lettre ce qui circule ou non entre leur corpus (livres, idées, étonnements communs), soumis parfois à la nécessité " nouvelle " de mieux se comprendre de part et d'autre, de comprendre à part égale ces doubles coups de force que sont le discours autorisé de la philosophie et les effusions souveraines et menaçantes de la littérature. Cependant, la tentation est grande de dramatiser cette conjonction " et ", en jouant de toutes les disjonctions qu'elle recèle. Si bien que la problématique prend parfois l'allure suivante: comment renverser la philosophie ou la faire se déborder, comment contrôler ou renverser ce qui toujours renverse par quelques excès déraisonnables ? Contre cette dernière manière un peu carrée de se poser la question, les quatre essais de ce collectif Philosophie et littérature - issu d'un Colloque tenu en juin 1978 à l'Univesité Western Ontario proposent plutôt "un projet général de confusion du philosophique et du littéraire ", en s'allouant avec bonheur toute la noblesse étymologique de cette confusion.

"Sparadrap » de Pierre Gravel amorce un retour au poète tragique Sophocle, contre son exclusion platonicienne prononcée dans la République. Style haut en couleurs qui se veut tragique, qui se déployait au même moment dans « D'un cerf à la robe tachetée et de quelques oiseaux. Essai sur l'indétermination de toute violence ", issu du Colloque " tragique et tragédie » et d'une longue recherche menée conjointement par le Département de Philosophie et le Programme de littérature comparée de l'Université de Montréal (Études françaises, XV, 1979, pp. 99-120). " «Molière et l'effet de psycha- 
nalyse » de Max Vernet propose avec brio une lecture de L'École des Femmes "temps du doute hyperbolique de Molière " : mise en scène et traduction du jeu d'Arnolphe, philosophe à qui échappe constamment sa philosophie ; plaisir de l'auteur à y restituer la " théorie cloacale " par le jeu des correspondances avec la Méditation seconde de Descartes. Plus classique, la longue "Communication romanesque et communication philosophique " de Guy Bouchard fait l'état de la question dans la foulée de la sémiologie littéraire et interroge avec beaucoup de précautions méthodologiques le statut du texte philosophique, s'il s'avère qu'il y a aussi en philosophie un "dédoublement mimétique du processus de communication » (entretien, dialogue, adresse au lecteur . . . impliquant narrateur, personnages, procédés référentiels). Enfin, ce collectif s'est vu augmenté de la version française de « Discursive criticism and epistemology " de Timothy J. Reiss (issu du collectif The Interpretation of Narrative, Toronto, University of Toronto Press, 1978) ; également en version française, une communication (Budapest, 1976) sur " Le principe d'indéterminisme et la critique du discours " qui développe quelques réflexions très pertinentes sur l'acte d'interprétation expérimentalement situé entre le discours critique et le texte critiqué, va et vient qui annonce aussi Logics of Literature pour le futur plaisir du lecteur.

De son propre aveu, Philosophie et littérature se rapporte à l'usage confondant d'une conjonction, mais en dérogeant à son propre programme : les deux premiers textes chargent contre une certaine caricature (facile) de la rationalité alors que le troisième maintient la disjonction, côté philosophie. C'est donc davantage par des effets de lecture transformés par certains effets d'écriture (d'ailleurs, inégalement distribués entre ces quatre essais) qu'advient ici la problématique entre philosophie et littérature. Ajoutant, par la référence à des disciplines déjà constituées et par la confusion institutionnelle du sujet, ajoutant donc un problème là où celui-ci est peut-être somme toute déjà réglé. Situation renversante. Mais pouvait-il en être autrement ? Bref, si le corpus étudié (Sophocle, Molière, exemples de la littérature romanesque) a déjà réglé la figure problématique que lui prêtent le regard critique ou la philosophie par le chevauchement synoptique entre les genres et les traditions de lecture, de quel lieu confondant s'amorce alors cette question ? Plus précisément, comment faire l'épreuve de cette conjonction tout en faisant comme si elle n'existait pas, ou comme si elle n'existait plus parce qu'on a produit un " nouveau " texte, l'autre confus et néanmoins diffus par le même?

Si le lecteur n'a pas de réponse immédiate à cette question - sans doute non pertinente en ce lieu - il ne peut se rabattre sur d'autres orientations possibles. Et c'est là la seule limite de ce collectif. Certes, dans son Avantpropos, Pierre Gravel situe son projet, présente l'écart qui permet aux différents collaborateurs d'habiter de diverses manières l'espace de la question. Cependant il est déplorable que la trame ethnophilosophique d'une telle problématique ne soit pas exposée, ni dans une perspective historique qui dépasserait de beaucoup le moment hégélien tel quel (songeons à la complexité du corpus étudié et aux recherches méta-philologiques de Peter Szondi, qui s'est donné la mort en 1971), ni dans la fine multiplicité de ses thèmes 
et de ses interrogations (songeons depuis la dernière décade, à toute la littérature de l'aphorisme, qui mériterait d'être repensée), ni dans la localisation territoriale d'un intérêt québécois à penser et à faire advenir ces rapports entre phịlosophie et littérature - par l'intermédiaire des poètes écrivants et de leurs lectures en philosophie, par l'intermédiaire des philosophes et de leurs proximité (citationnelle ou non) à l'expérience poétique ou aux plaisirs de l'écriture. Sont félicités dans cet Avant-propos Jean-Luc Nancy et Philippe Lacoue-Labarthe pour leur intérêt au romantisme allemand, Michel Serres sur le même et l'autre, qui " a dit là-dessus ce qu'il fallait "; mentionné enfin, le numéro spécial d'Études littéraires (IX, 1976) sur "Littérature et philosophie " - pour l'article de Guy Bouchard du même titre, alors que n'est pas relevé l'essai de Jean-Paul Brodeur ("Quelques remarques sur la théorie des récits ", pp. 525-553) qui commence avec deux fictions narratives (une au masculin et l'autre au féminin) calquées sur la dialectique du maître et de l'esclave et se termine, contre le spectre de la tautologie, par un effet de surprise.

Afin de mettre en place une filière entre philosophie et littérature et permettre à un autre lecteur de conjuguer la problématique de ce livre (19781979) avec une histoire qui le concernera de toute manière, je donne ici quelques indices complémentaires. Le numéro spécial « Littérature et philosophie mêlées " de Poétique $(21,1975)$ avec Michel Deguy sur le besoin de connaissance confuse, Jean Starobinski, Jean-Luc Nancy et Philippe LacoueLabarthe, Jacques Derrida, un important document sur " le dialogue des genres ». Le double numéro spécial «Graphesis : Pespectives in Literature and Philosophy " de Yale French Studies $(52,1975)$ qui s'ouvre avec une épigraphe de John Ashberry Drame Bourgeois ( Your emptying glance, prisms which I treasure up ») et qui regroupe entre autres Marie-Rose Logan, Paul de Man, Shoshana Felman ; en traduction in-édite, Jacques Derrida, JeanFrançois Lyotard, Michel Serres; avec une section très révélatrice "Three American Perspectives " (pp. 229-287) — quand pourrons-nous enfin inscrire "Perspectives québécoises" sur et autour de la conjonction géographique "Philosophie française et civilisation française aux U.S.A. ", ou encore "Philosophies française et américaine et culture québécoise " ? Urgence collective à décréter, pour le progrès de notre conscience philosophique toujours étrangement stéréo-typée. Par la même occasion, repensant au palabre territorial de Julian Marias « Les genres littéraires en philosophie » dans Revue Internationale de philosophie, 90 (1969) ; numéro spécial portant sur « La communication en philosophie ", avec une belle auto-critique (argumentée) de $\mathrm{H}$. W. Johnstone Jr. Donc recueillir à chaque session académique autant Glyph que la New Literary History (et récemment en ce lieu, «Philosophy as a Kind of Writing : an Essay on Derrida ", X, 1978, pp. 141-160 ; article de Richard Rorty, important de par les présupposés géographiques de sa perception) sans oublier cette revue plus classique Philosopby and Literature fondée en 1976 à l'Université du Miçhigan (Dearborn, Michigan 48128), qui réserve beaucoup de surprise au lecteur quant au domaine littéraire anglosaxon. 
Et côté langue poétique maintenant, ce qui manque étrangement à ce collectif ? En attendant qu'un archiviste (québécois ou non) se penche avec amour sur les rapports entre philosophie (québécoise ou non) et poésie québécoise à la Nouvelle Barre du jour et aux refoulées Herbes rouges (ou encore à l'Estuaire, puisque ce phénomène n'est pas «Montréaliste »), régressant dans le temps jusqu'au premier numéro de La Relève (1934) et de Gants du ciel (1943) avec les "Allégories " de Gilles Hénault, passant par la « Note sur le langage philosophique " du poète et ex-professeur d'Esthétique en philosophie Jacques Brault (premier Dialogue, 1, 1962, pp. 51-55; où se retrouvent également deux philosophes exilés par des conjonctions extrêmes, LouisB. Geiger et Hugues Leblanc), méditer de l'intérieur de leur tradition française et mallarméenne l'invention poétique de cet écart qu'ils ont eux-mêmes par eux-mêmes pensé (la philosophie française ne serait-elle pas au fond un accident de parcours dans le poème d'une langue souveraine ?) : explorer par conséquent Yves Bonnefoy, Edmond Jabès, Roger Munier, Jacques Garelli, Lorand Gaspar, l'expérience singulière d'un Philippe Jaccottet, la lecture bouche ouverte d'un Pascal Guignard ou de Michel Deguy, poète et exprofesseur de philosophie qui a déjà sillonné la terre québécoise sur un mode discret, "La poésie en questions ", Revue des Sciences philosophiques et théologiques, LVI (1972) pp. 19-42 ; ou d'une autre génération, Claude RoyetJournoud qui a composé le collectif "Travail de Poésie ", Revue de l'Université de Bruxelles, 1-2 (1979). Ruse d'un feed-back effectif : quel est désormais (ou depuis toujours) la philosophie de notre littérature, la pensée et l'algorithme géographique de notre langue?

Autant de manières de situer à la lettre la permanence d'une problématique, de travailler sur un autre mode de réjouissance le lieu ou le non lieu d'une conjonction, chacun soumis à la nécessité de comprendre ce double coup de force réciproque entre philosophie et littérature, qui parfois s'institutionnalise en institutionnalisant certaines topiques au détriment d'autres topiques. Pourquoi philosophes et littéraires chercheraient-ils alors à mettre en scène la raison d'un lieu - qui demeure comme un point de rencontre momentané - s'il ne devient pas évident que cette raison se déploie à la surface de l'espace disciplinaire qui en permet la conjonction, ou demeure suspendue dans l'innommable effacement de ce même espace, seul prélude à la pensée.

Au cœur de la jonction originelle, tout s'exprime par le corps qui l'écrit sans cesse pour aussitôt la dévorer comme un chaînon manquant.

Département de philosophie

Collège de Maisonneuve 